\title{
ESTIMACIÓN DE LA FUNCIÓN AMBIENTAL DE LOS PAISAJES BOSCOSOS Y ESTEPARIOS DE TIERRA DEL FUEGO A TRAVÉS DE LA CARACTERIZACIÓN DE SU AVIFAUNA USANDO DATOS DE EBIRD
}

\author{
Benítez, Julieta ${ }^{1}$; Huertas Herrera, Alejandro²; Martínez Pastur, Guillermo³; \\ Pizarro, J. Cristóbal ${ }^{4}$; Lencinas, María Vanessa ${ }^{5}$
}

\section{RESUMEN}

Las funciones ambientales de los bosques son afectadas por las actividades productivas y turístico-recreativas que en ellos se realizan, siendo las aves buenos y versátiles indicadores de cambio ambiental. En la Isla Grande de Tierra del Fuego (Argentina), los paisajes boscosos (40\% de la superficie en el centro-sur de la Isla) y esteparios (25\%, predominantemente en el norte) son los mejor representados, mientras que las aves son los vertebrados más abundantes y diversos que la habitan.

El objetivo de este trabajo fue evaluar la utilidad de la base de datos provista por la plataforma global eBird ${ }^{6}$ para estimar la función ambiental de conservación del hábitat natural y de la diversidad biológica de los paisajes boscosos y esteparios de Tierra del Fuego, a través de la caracterización de su avifauna. Usando eBird se recopiló información sobre observaciones de aves (latitud y longitud, fecha de observación y especies) desde 1973 hasta febrero del 2016.

Excluyendo las especies de aves exclusivamente costeras, marinas y dulceacuícolas, la información se resumió en datos mensuales por año de cada especie para cada "localidad" (coordenada geográfica en latitud/longitud), evitando la repetición de observaciones de una misma especie para un determinado punto. Los datos de latitud y longitud se geoprocesaron en una plataforma SIG para determinar el paisaje dominante en cada localidad, clasificándolo en una de cuatro categorías: estepa, bosque de ñire, bosque de lenga y bosque mixto.

Se evaluaron cantidad de observaciones, de localidades y de especies en general y para cada categoría, identificando las aves raras o comunes y las más frecuentes en cada paisaje. Asimismo, se identificaron las especies compartidas por todos los paisajes y las exclusivas. Por último, se detectaron los gremios alimenticios más comunes y el número de observaciones de las especies con funciones ecológicas específicas para cada paisaje.

La base de datos permitió extraer información sobre 77 especies y 7.984 observaciones en 493 localidades. Se detectaron 44 especies (56\%) compartidas en todos los paisajes (ej. Carduelis barbata, Falco sparverius y Vultur gryphus) y 11 (14\%) raras (ej. Asthenes pyrrholeuca, Muscisaxicola maculirostris y Phrygilus fruticeti), varias de las cuales se observan circunstancialmente en la Isla pero cuya distribución típica no la incluye.

Respecto de la cantidad de observaciones, el $66 \%$ se realizó en paisajes dominados por bosques mixtos ( $42 \%$ de las localidades), los cuales incluyeron gran cantidad de observaciones en

\footnotetext{
${ }^{1}$ Lic. en Ciencias Ambientales, CONICET, Ushuaia, Argentina, jbenitezgaias@gmail.com

2 Ecólogo (Mag.), Ushuaia, Argentina, alejandrohuertasherrera@gmail.com

${ }^{3}$ Ing. Forestal (Mag.) Dr., CONICET, Ushuaia, Argentina, gpastur@conicet.gov.ar

${ }^{4}$ Med. Veterinario (Mag.), Dr., CONICET, Ushuaia, Argentina, jcpizarrop@gmail.com

5 Ing. Forestal, Dra., CONICET, Ushuaia, Argentina, mvlencinas@conicet.gov.ar

${ }^{6}$ http://ebird.org/
} 
el Parque Nacional Tierra del Fuego y en la ciudad de Ushuaia, mientras que los otros paisajes solo incluyeron $7-17 \%$ de las observaciones (13-23\% de las localidades). En cuanto a la riqueza específica, fue mucho mayor en paisajes con predominio de bosque mixto (74 especies) que en los otros paisajes (estepa=59, bosque de ñire y lenga $=55$ en cada uno), siendo probablemente subestimada en estos por la baja cantidad de observaciones y localidades. Las especies exclusivas fueron 7 en los paisajes con bosque mixto y 3 en estepa, mientras que no se observó ninguna en paisajes dominados por ñire o lenga.

Las aves más frecuentes en cada paisaje se encuentran entre las más conspicuas y conocidas y gran cantidad de ellas coincidieron entre paisajes (ej., Chloephaga picta en estepa, ñire y mixto, Zonotrichia capensis en ñire, lenga y mixto, Milvago chimango en lenga y mixto).

Todos los gremios y especies con funciones ecológicas específicas presentaron mayor número de observaciones en los bosques mixtos. Aunque eBird permitió encontrar diferencias y similitudes entre paisajes, se observa cierta tendencia espacial de concentración de las observaciones, lo que indicaría que son necesarios estudios mejor distribuidos espacialmente para caracterizar la avifauna y concluir sobre la función ambiental de los paisajes analizados.

Palabras clave: Avifauna, Tierra del Fuego, Argentina

\section{SUMMARY}

The environmental function of forest is affected by agro-forestry and tourism-recreational activities, being birds good and versatile ecological indicators of environmental changes. In Isla Grande of Tierra del Fuego (Argentina), forests (40\% of the surface in the center-south of the island) and steppes (25\%, predominantly in the north) are the most represented ecosystems, while birds are the most abundant and diverse vertebrates that inhabit it.

The aim of this study was to evaluate the usefulness of the database provided by the international platform eBird ${ }^{7}$ to estimate the environmental function of forests and steppes on Tierra del Fuego through the characterization of its birdlife. Using the eBird database, information of bird observations (latitude and longitude, date of observation and species) from 1973 to February 2016 was collected.

Observations exclusively coastal and marine birds were excluded. Information was summarized to monthly data for each species per year per "locality" (geographical coordinate in latitude/longitude), avoiding the repetition of observations of the same species for a given point. Latitude and longitude data were geoprocessed on a GIS platform to determine the dominant landscape in each locality, classifying each point in one of four categories: steppe, Nire forest, Lenga forest and mixed forest.

The number of observations, locality and species in general and in each category, were evaluated identifying the rare species or common and frequent birds in each landscape. Likewise, those shared among landscapes and the exclusive ones, were identified and analyzed. Finally, the most common food guilds and the number of observations of species with specific ecological functions for each category were detected.

The database allows extract information about 77 species and 7,984 observations on 493

\footnotetext{
${ }^{7}$ (http://ebird.org/)
} 
localities. It was possible to detect 44 species (56\%) shared among all landscapes (eg. Carduelis barbata, Falco sparverius y Vultur gryphus) and 11 (14\%) rare (eg. Asthenes pyrrholeuca, Muscisaxicola maculirostris y Phrygilus fruticeti), several of which are observed on the island circumstantially but whose typical distribution does not include it. Respect of the number of observations, $66 \%$ was in landscapes dominated by mixed forests (42\% of the locality), which included larger number of observations in the Tierra del Fuego National Park and in Ushuaia city, while other landscapes included only $7-17 \%$ of observations $(13-23 \%$ of the locality).

In terms of species richness, it was much higher in mixed forest (74 species) than in the other landscapes (steppe $=59$, Lenga and Nire forest $=55$ in each), probably being underestimated by the low number of observations and locality. The exclusive species were 7 in mixed forest and 3 in steepe, while none was seen in landscapes dominated by ñire or lenga forests.

The most frequent birds in each landscape are among the most conspicuous and best known and most of them are shared among landscapes (eg, Chloephaga picta in steppe, Nire and mixed forest, Zonotrichia capensis in ñire, lenga and mixed forest, Milvago chimango in Lenga and mixed forest).

All the guilds and species with specific ecological functions presented greater number of observations in the mixed forests. Although eBird allowed finding differences and similarities between landscapes, there are trends in the observations, suggesting that more studies are needed to characterize the avifauna and conclude about environmental function of the analyzed landscapes, which must be better spatially distributed.

Keywords: Birdlife, Tierra del Fuego, Argentina

\section{INTRODUCCIÓN}

La preocupación por la conservación y monitoreo de la biodiversidad ha motivado en el mundo entero múltiples esfuerzos para generar bases de datos para la recopilación de información sobre presencia, distribución y abundancia de especies para un tiempo y lugar determinado. Aunque se ha avanzado en el desarrollo de redes de sensores remotos autónomos para tales fines (Hochachka et al., 2007). La mayoría de los datos sobre biodiversidad a nivel de especie son colectados por los seres humanos a través de sistemas o plataformas para su recopilación y análisis a gran escala (Kelling, 2008). Las ciberestructuras, plataformas sincronizadas en línea, han emergido fuertemente en los últimos años y han permitido la participación ciudadana en la colecta, síntesis y análisis de datos sobre biodiversidad (Michener et al., 2001).

La plataforma eBird ${ }^{8}$ es un proyecto lanzado por el Laboratorio Cornell de Ornitología y el Instituto Nacional Audubon Society en el 2002, que involucra una red mundial de observadores de aves (ciudadanos-científicos) para reportar observaciones usando protocolos estandarizados a través de internet (Sullivan et al., 2009). EBird recopila información sobre la distribución y abundancia de aves, aprovechando la enorme popularidad de observar aves para crear una red global de voluntarios que envían sus observaciones de aves a través de Internet a un repositorio central de datos (Sullivan et al., 2014).

Las aves son observadas y disfrutadas por las personas todos los días debido a que existen casi 10.000 especies que ocupan casi todos los ambientes terrestres y acuáticos y porque

\footnotetext{
${ }^{8} \mathrm{http}: / /$ www.ebird.org
} 
están vinculadas a procesos bióticos en muchos niveles. Además, las aves, están entre los grupos de animales más estudiados, y son en su mayoría diurnas, morfológicamente conspicuas y abundantes, siendo así fácilmente observables por las personas (Gill, 2006).

De esta forma, a través de esta herramienta, los ornitólogos y observadores de aves pueden organizar y difundir observaciones de aves y promover el birdwatching como una actividad recreativa que apoya el estudio y conservación de la biodiversidad. Al mismo tiempo, estos datos proporcionan a los científicos información que puede ser valiosa para mejorar el entendimiento de la presencia de las especies de aves, sus tiempos de migración y, en algunos casos, su abundancia relativa en una variedad de escalas espaciales y temporales (Sullivan et al., 2009), particularmente en sitios remotos y poco estudiados.

En este sentido, la porción argentina de Isla Grande de Tierra del Fuego (TDF, Figura $\mathrm{N}^{\circ} 1$ ), es un sitio remoto donde la necesidad de generar redes y participación ciudadana para la recopilación de datos es mayor. Debido a que es un sitio de colonización relativamente reciente, tiene muy poca población y presenta importantes dificultades climáticas y topográficas como para realizar colectas de datos lideradas por especialistas, uniformemente distribuidas en espacio y tiempo. En TDF las aves son los vertebrados más diversos y abundantes (Lencinas et al., 2005). Los paisajes son dominados por bosques en el centro-sur ( $40 \%$ de su superficie) y por la estepa al norte $(25 \%)$. El $35 \%$ restante corresponde a paisajes de vegetación de altura, cuerpos de agua y grandes extensiones de turbales.

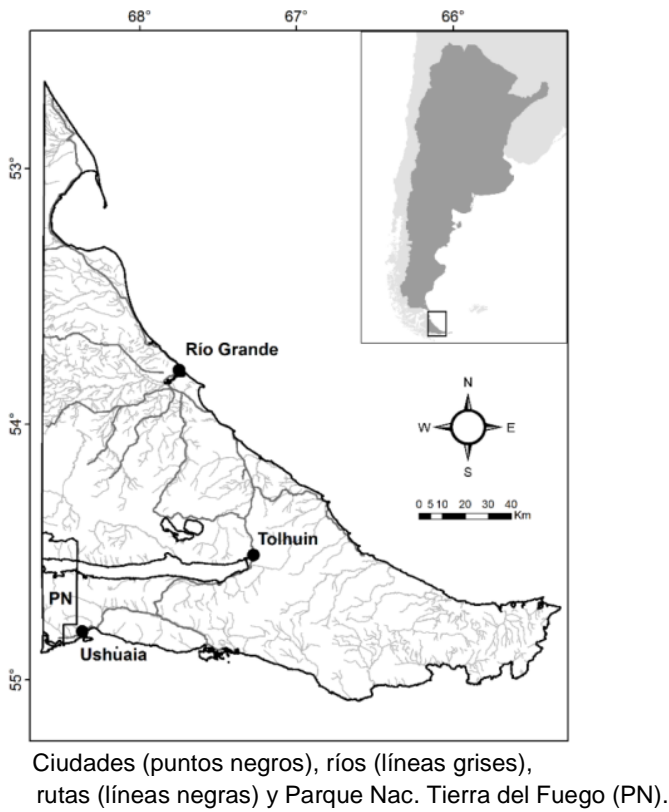

Figura $\mathbf{N}^{\circ} 1$

CARACTERIZACIÓN DE LA PROVINCIA DE TIERRA DEL FUEGO 
En TDF se han hecho estudios a nivel de parche para entender la comunidad de aves con respecto a su dinámica en bosques mixtos de lenga y de ñire. Tanto en la parte argentina del archipiélago (Martínez Pastur et al., 2015) como para isla Navarino en Chile (Ippi et al., 2009), se han establecido relaciones entre dichas comunidades y variables como la estructura forestal y la estacionalidad. En los bosques templados de Chile, Meynard y Quinn (2008) encontraron que los gradientes ambientales influyen en la estructura espacial, siendo el clima y el tamaño de los fragmentos factores indirectos que explican la diversidad de aves, a través de los cambios en la estructura vegetal. Por otra parte, hay investigaciones que abarcaron las dinámicas de aves que habitan los humedales (Ibarra et al., 2010) y los que vinculan los ambientes marino-costeros con el bosque (Pizarro et al., 2012).

Las funciones principales de los bosques pueden agruparse en protectoras y ambientales (Gottle y Sène, 1997) (ej., protección de los recursos de agua y del suelo, atenuación del clima local y reducción del impacto de emisiones de gases) y estas pueden verse afectadas por las actividades agroforestales y turístico-recreativas que en ellos se realizan. Entre estas, la conservación del hábitat natural y de la diversidad biológica es una de las más relevantes, siendo las aves buenos y versátiles indicadores ecológicos por ser los vertebrados más abundantes y diversos en TDF (Lencinas et al., 2005). Además, en estas latitudes, las aves ocupan muchos nichos y papeles ecológicos claves (Díaz et al., 2005). Por ejemplo, se ha demostrado que varias especies cumplen funciones ecológicas específicas, como polinizadoras (ej. Elaenia albiceps), dispersoras de semillas (ej. de Berberis buxifolia-calafate por Turdus falcklandii), o alimento de rapaces (Aphrastura spinicauda o Troglodytes aedon) (Humphrey et al., 1970). El análisis de la distribución de las aves, así como de las funciones ecológicas específicas que cumplen en los distintos ambientes boscosos, podría brindar información sobre el estado de conservación del hábitat natural y su diversidad biológica, en forma comparativa con ambientes esteparios no boscosos de TDF.

En sitios remotos como TDF, plataformas como eBird, que involucra a una red mundial, en este caso de observadores de aves, podrían ser de gran utilidad para el estudio de funciones ambientales de los paisajes. Estas funciones, como la conservación del hábitat natural y de la biodiversidad, pueden variar de acuerdo a los diferentes paisajes boscosos y esteparios y a las actividades productivas y turístico-recreativas que en ellos se realizan, lo que hace necesario su estimación y posterior comparación.

\section{OBJETIVOS}

El objetivo de este trabajo fue evaluar la utilidad de la base de datos provista por la plataforma internacional eBird para estimar la función ambiental de conservación del hábitat natural y de la diversidad biológica (riqueza y distribución de especies, funciones ecológicas específicas) de los paisajes boscosos y esteparios de Tierra del Fuego, a través de la caracterización de su avifauna.

\section{MATERIAL Y MÉTODO}

En este trabajo se utilizó de la base de datos eBird información recopilada desde 1973 hasta febrero del 2016 para el sector argentino de la Isla Grande de TDF. Los árboles dominantes en los paisajes boscosos de TDF son la lenga (Nothofagus pumilio), el ñire ( $N$. antarctica) y el guindo ( $N$. betuloides), ocupando las dos primeras el $44 \%$ y $25 \%$ de la superficie forestal como masas puras, respectivamente, siendo el resto bosques mixtos de lenga y guindo (Moore, 1983). 
Los bosques de $N$. pumilio son ecosistemas altamente valiosos a nivel mundial, que se caracterizan por constituir sistemas forestales simples adecuados para una gran gama de estudios (Lencinas et al., 2004). Solo en la provincia argentina de Tierra del Fuego (TDF), los bosques de lenga abarcan alrededor de 317 mil hectáreas (Collado, 2001). Los bosques de $N$. antarctica, por otro lado, ocupan alrededor de 181 mil hectáreas en TDF (Collado, 2001) y se encuentran al sur de la zona donde predomina la estepa. El ñire ocurre mayormente en un paisaje donde los parches de bosque se entremezclan con pastizales, arbustales y turbales. Es un árbol cuyo desarrollo de ramas es muy intrincado desde el suelo y de altura menor que la de sus congéneres de TDF, de una asombrosa plasticidad ecológica.

La estepa magallánica abarca la parte norte de la isla siendo su límite Sur una línea que corre de $\mathrm{O}$ a $\mathrm{E}$, desde el límite con Chile, sobre el Río Grande hasta el litoral Atlántico, con una superficie de $4.180 \mathrm{~km}^{2}$. De relieve ondulado, esta zona presenta cañadones que limitan planicies bajas y húmedas ("vegas") con abundante vegetación herbácea compuesta por diferentes especies de gramíneas (Festuca spp., Poa spp., Bromus spp., Agrostis spp.), dicotiledóneas (Taraxacum officinale, Caltha sagittata), ciperáceas (Carex spp.) y juncáceas (Juncus spp., Luzula spp.). Las tierras altas se encuentran cubiertas, generalmente, por plantas de "coirón dulce" (Festuca gracillima), "mata negra" (Chiliotrichum difussum), y la "murtilla" (Empetrum rubrum) (Bonino y Sbriller, 1991; Montes et al., 2000).

El denominado bosque mixto magallánico (Promis et al., 2008) presenta características singulares por incluir al guindo, especie perennifolia acompañante del bosque de lenga, y con participación en distintas proporciones de otras especies de hoja ancha y perenne como Drimys winteri (canelo), Maytenus magellanica (leña dura) y Embothrium coccineum (notro). La presencia del guindo se torna cada vez más evidente hasta hacerse dominante a medida que se acerca a la costa del canal de Beagle, a favor de mayores precipitaciones y menores oscilaciones térmicas. También puede dominar en algunas ubicaciones cercanas a grandes masas de agua interiores, como es el caso de los márgenes del Lago Fagnano, que reproducen a menor escala las citadas condiciones climáticas (Moore, 1983; Promis et al., 2008).

Para registrar observaciones de aves, los usuarios registrados en el portal web de eBird deben ingresar a una plataforma e indicar la hora, fecha de conteo y método de avistamiento (punto de conteo, caminata o transecto), esfuerzo (duración de la observación o distancia recorrida) y la localidad o ubicación geográfica del lugar a través de sus coordenadas geográficas o su ubicación espacial en un mapa online (google maps). Para los métodos de observación, eBird ofrece varias opciones, incluyendo además avistamientos incidentales e históricos. Las especies son ingresadas sobre una lista de especies ordenadas en orden taxonómico o por frecuencia de avistamiento. La plataforma posee además filtros automatizados de calidad de datos desarrollados por expertos regionales en aves que, en la mayoría de los casos, revisan los reportes de especies consideradas raras para el lugar y/o la época. Los datos son públicos y se almacenan en tiempo real a través del sitio web eBird u otras aplicaciones desarrolladas por la plataforma.

Para este trabajo, de cada observación registrada en eBird para TDF, se tomaron los datos de la localidad (coordenadas geográficas en latitud/longitud), la fecha de observación y las especies registradas. La información se resumió a datos mensuales por año de cada especie para cada localidad, evitando así la repetición de observaciones para la misma especie y ubicación. Se excluyeron las observaciones de aves costeras, marinas y dulceacuícolas. Complementariamente se utilizó una plataforma SIG para visualizar las localidades por cada especie y, posteriormente, mediante un mapa de tipos de vegetación, se determinó el tipo de paisaje predominante en cuatro categorías: estepa, bosque de ñire, bosque de lenga y bosque mixto (Figura $\mathrm{N}^{\circ} 2$ ). Cabe mencionar 
que, dentro de la localidad clasificada según el tipo de paisaje predominante, pueden existir pequeñas superficies con otros tipos de vegetación (por ej., pequeñas áreas de pastizal inmersas en una matriz paisajística de bosque de lenga). Luego, para cada categoría de paisaje se contabilizaron la cantidad de observaciones, de localidades, y de especies registradas.

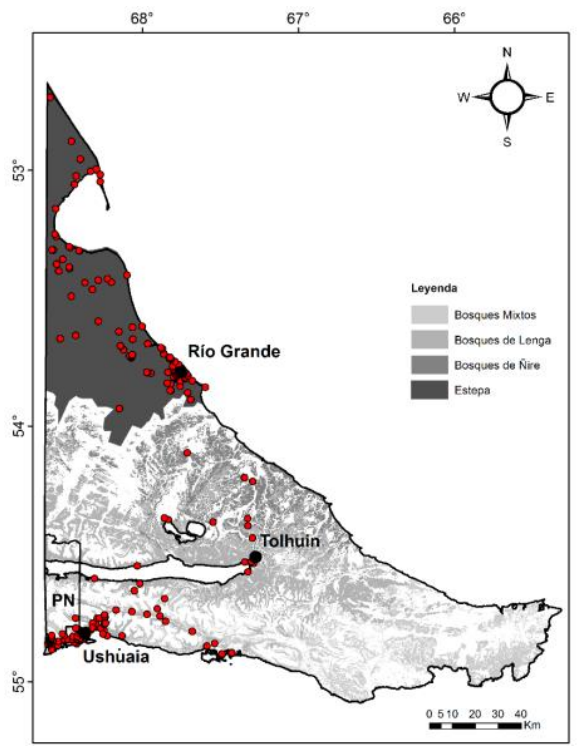

Ciudades (puntos negros), Parque Nac. TDF (PN) y localidades (puntos rojos)

Figura $\mathrm{N}^{\circ} 2$

CATEGORÍAS DE PAISAJE (Collado, 2001) EN LA ISLA GRANDE DE TIERRA DEL FUEGO (ARGENTINA) TOMADOS DE LA BASE DE DATOS DE eBIRD (1973-2016).

Posteriormente, las especies se clasificaron taxonómicamente, por gremio alimenticio (Pizarro et al., 2012) y como raras (detectadas solamente en 1 o 2 observaciones) o comunes. Asimismo, se analizó si eran compartidas entre todas las categorías de paisaje (especies con al menos una observación en las cuatro categorías), compartidas entre dos o tres tipos de paisaje (bosques en forma conjunta vs. estepa, y ñire vs. lenga vs. guindo), o exclusivas (especies que solo se observaron en una categoría). Luego, se identificaron las especies de aves más frecuentes (avistadas en mayor cantidad de localidades), en general y en cada categoría de paisaje. Finalmente, se agruparon las especies de un mismo gremio alimenticio y se determinó el total de observaciones de individuos pertenecientes a cada gremio en general y en cada categoría de paisaje.

Además, se identificaron especies con funciones ecológicas específicas (ej. polinizadores, dispersores de semilla) y se contabilizó el número de observaciones de estas especies en cada paisaje. 


\section{RESULTADOS}

La base de datos permitió extraer información de 7.984 observaciones en 493 localidades. El $66 \%$ de las observaciones se realizó en paisajes dominados por bosques mixtos (42\% de las localidades), principalmente en el Parque Nacional TDF y la ciudad de Ushuaia. Los otros tipos de paisajes representaron solo el $7-17 \%$ de las observaciones y el $13-23 \%$ de las localidades (Figura $\mathrm{N}^{\circ} 3$ ).
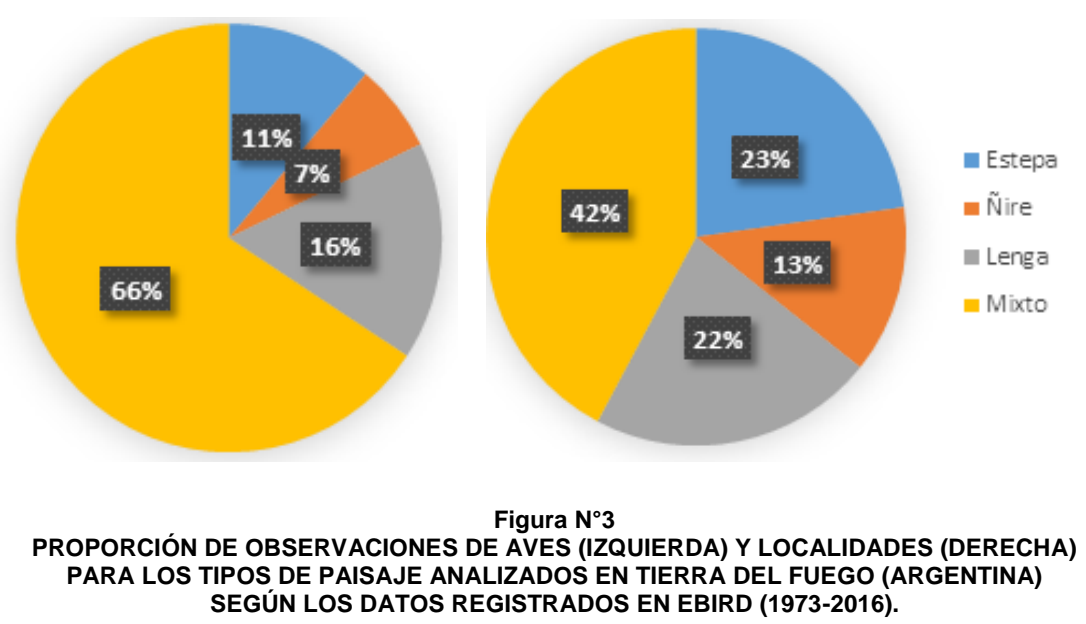

De esta base de datos, se identificaron 77 especies (Cuadro $N^{\circ} 1$ ), pertenecientes a 12 órdenes y 26 familias. Entre ellas, 55 especies fueron Paseriformes, siete Falconiformes, cinco Accipitriformes y cinco Strigiformes, tres Columbiformes, dos Cathartiformes y dos Piciformes, y se encontró solo una única especie de los géneros Anseriformes, Apodiformes, Charadriiformes, Pelecaniformes y Psittaciformes. Del total de especies registradas, once (14\%) fueron consideradas raras, mientras que el resto de las especies (66) fueron comunes. Entre estas, solo 28 especies se identificaron en más de 100 observaciones.

La riqueza específica más alta fue registrada en paisajes con predominio de bosque mixto (74 especies, $96 \%$ del total de especies) que en los otros paisajes (estepa $=59$ especies, bosque de ñire y lenga $=55$ especies en cada uno). El bosque mixto fue el ambiente que compartió mayor número de especies con el resto de los ambientes ( 56 con la estepa y 55 con bosque de ñire y lenga) (Figura № 4).

Por otra parte, $44(56 \%)$ especies fueron compartidas entre todas las categorías de paisajes analizados, siendo $C$. picta, $M$. chimango y $Z$. capensis las especies mejor representadas (con 546, 506, y 451 observaciones) (Figura $N^{\circ} 4$ ). Asimismo, sólo fueron observadas especies exclusivas en los paisajes dominados por bosques mixtos (siete especies) y estepa (tres especies) (Cuadro $\mathrm{N}^{\circ} 2$ ). Todas las especies exclusivas del bosque mixto y una de la estepa fueron especies que se consideraron raras. 


\section{Cuadro $\mathrm{N}^{\circ} 1$}

CLASIFICACIÓN TAXONÓMICA, NOMBRE COMÚN Y GREMIO ALIMENTICIO DE LAS ESPECIES CON MÁS DE 100 OBSERVACIONES EN LOS REGISTROS DE EBIRD (1973-2016) DE TIERRA DEL FUEGO

(ARGENTINA)

\begin{tabular}{|c|c|c|c|c|}
\hline Orden & Familia & Especie & Nombre Común & $\begin{array}{c}\text { Gremio } \\
\text { Alimenticio }\end{array}$ \\
\hline Accipitriformes & Accipitridae & Geranoaetus melanoleucus & Águila mora & $\mathrm{CC}$ \\
\hline Anseriformes & Anatidae & Chloephaga picta & Cauquén común & $\mathrm{H}$ \\
\hline Cathartiformes & Cathartidae & Cathartes aura & Jote cabeza colorada & $\mathrm{Ca}$ \\
\hline Cathartiformes & Cathartidae & Vultur gryphus & Cóndor andino & $\mathrm{Ca}$ \\
\hline Charadriiformes & Charadriidae & Vanellus chilensis & Tero común & 1 \\
\hline Falconiformes & Falconidae & Caracara plancus & Carancho & $\mathrm{CC}$ \\
\hline Falconiformes & Falconidae & Milvago chimango & Chimango & $\mathrm{O}$ \\
\hline Falconiformes & Falconidae & Phalcoboenus albogularis & Matamico blanco & $\mathrm{Ca}$ \\
\hline Passeriformes & Emberizidae & Phrygilus patagonicus & Comesebo patagónico & $\mathrm{O}$ \\
\hline Passeriformes & Emberizidae & Zonotrichia capensis & Chingolo & $\mathrm{O}$ \\
\hline Passeriformes & Fringillidae & Carduelis barbata & Cabecitanegra austral & $\mathrm{G}$ \\
\hline Passeriformes & Furnariidae & Aphrastura spinicauda & Rayadito & 1 \\
\hline Passeriformes & Furnariidae & Cinclodes patagonicus & Remolinera araucana & $\mathrm{C}$ \\
\hline Passeriformes & Furnariidae & Pygarrhichas albogularis & Picolezna patagónico & 1 \\
\hline Passeriformes & Hirundinidae & Tachycineta meyeni & Golondrina patagónica & 1 \\
\hline Passeriformes & Icteridae & Curaeus curaeus & Tordo patagónico & $\mathrm{O}$ \\
\hline Passeriformes & Icteridae & Sturnella loyca & Loica común & $\mathrm{O}$ \\
\hline Passeriformes & Passeridae & Passer domesticus & Gorrión & $\mathrm{O}$ \\
\hline Passeriformes & Troglodytidae & Troglodytes aedon & Ratona común & 1 \\
\hline Passeriformes & Turdidae & Turdus falcklandii & Zorzal patagónico & $\mathrm{O}$ \\
\hline Passeriformes & Tyrannidae & Anairetes parulus & Cachudito pico negro & 1 \\
\hline Passeriformes & Tyrannidae & Elaenia albiceps & Fiofío silbón & $\mathrm{O}$ \\
\hline Passeriformes & Tyrannidae & Lessonia rufa & Sobrepuesto & I \\
\hline Passeriformes & Tyrannidae & Muscisaxicola maclovianus & Dormilona cara negra & 1 \\
\hline Passeriformes & Tyrannidae & Xolmis pyrope & Diucón & $\mathrm{O}$ \\
\hline Pelecaniformes & Threskiornithidae & Theristicus melanopis & Bandurria austral & $\mathrm{C}$ \\
\hline Piciformes & Picidae & Campephilus magellanicus & Carpintero gigante & 1 \\
\hline Psittaciformes & Psittacidae & Enicognathus ferrugineus & Cachaña & $\mathrm{G}$ \\
\hline
\end{tabular}

Gremios alimenticios: $\mathrm{C}=$ carnívoro, $\mathrm{Ca}=$ carroñero, $\mathrm{CC}=$ carnívoro y carroñero, $\mathrm{G}=$ granívoro, I=insectívoro, O=omnívoros y $\mathrm{H}=$ herbívoros. 

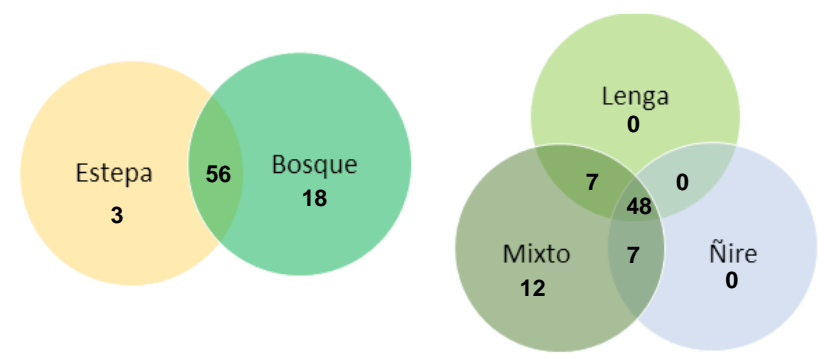

Comparando ambientes boscosos y no boscosos (considerando los bosques de ñire, lenga y mixtos en forma conjunta), y los tres tipos de bosques.

Figura $\mathrm{N}^{\circ} 4$

ESPECIES DE AVES COMPARTIDAS Y EXCLUSIVAS

ENTRE TIPOS DE PAISAJE DE TIERRA DEL FUEGO

\section{Cuadro $\mathrm{N}^{\circ} 2$}

ESPECIES EXCLUSIVAS DE AVES PARA LOS PAISAJES ANALIZADOS DE TIERRA DEL FUEGO EN BASE A DATOS REGISTRADOS EN EBIRD (1973-2016)

\begin{tabular}{|l|l|}
\hline \multicolumn{1}{|c|}{ Bosque mixto } & \multicolumn{1}{c|}{ Estepa } \\
\hline Agelaius thilius & Asthenes anthoides \\
\hline Anthus hellmayri & Asthenes pyrrholeuca \\
\hline Asthenes modesta & Falco femoralis \\
\hline Colaptes pitius & \\
\hline Hymenops perspicillatus & \\
\hline Metriopelia melanoptera & \\
\hline Muscisaxicola maculirostris & \\
\hline
\end{tabular}

Los bosques de ñire y de lenga no presentaron especies exclusivas

Las especies más frecuentes fueron $C$. picta, Z. capensis y $M$. chimango con 254,232 y 229 localidades, respectivamente. En la estepa, las especies observadas en mayor cantidad de localidades fueron $C$. picta, $S$. loyca y $L$. rufa; en el bosque de ñire fueron $Z$. capensis y Theristicus melanopis; y en el bosque de lenga, $M$. chimango y $Z$. capensis. En el bosque mixto las especies más frecuentes fueron $M$. chimango, $C$. picta y $Z$. capensis.

Los gremios más observados fueron omnívoros, insectívoros y carnívoros con 2.765, 2.097 y 1.009 observaciones, respectivamente. Todos los gremios alimenticios presentaron mayores observaciones en los bosques mixtos, y en segundo lugar en los bosques de lenga, a excepción de los carnívoros y del único herbívoro (C. picta) que se observaron más en bosques mixtos, pero en segundo lugar en la estepa.

Lo mismo ocurrió con las especies que presentan funciones ecológicas específicas como dispersoras de semilla (Enicognathus ferrugineus y $T$. falcklandii), polinizadoras (E. albiceps), o de limpieza del ecosistema ( $V$. gryphus), las cuales presentaron mayor número de observaciones en los bosques mixtos. 


\section{DISCUSIÓN}

Aunque eBird permitió encontrar diferencias y similitudes entre paisajes, se observa cierta tendencia en las observaciones. Por ejemplo, la riqueza específica fue mayor en paisajes con predominio de bosque mixto que en los otros paisajes, además, fue el ambiente que compartió mayor número de especies con el resto de los ambientes. Ambos resultados probablemente estén sobreestimados por la alta cantidad de observaciones y localidades en dicha categoría de paisaje, la cual incluyó al Parque Nacional Tierra del Fuego y a la ciudad de Ushuaia. Lo mismo ocurre con los grupos funcionales, ya que en paisajes esteparios se esperaría observar una mayor cantidad de individuos de especies granívoras (ej. E. ferrugineus y $T$. falcklandii). Sin embargo, esto no se observó en esta base de datos.

La distribución descrita en la bibliografía (Narosky e Yzurieta, 2010) para cinco de las especies consideradas raras (Agelaius thilius, Anthus hellmayri, Asthenes modesta, Hymenops perspicillatus y Muscisaxicola maculirostris) no incluye a TDF (además, no suelen habitar el tipo de paisaje donde se las registró), por lo que es probable que estos datos sean erróneos, indicando que es necesario mejorar la participación y los filtros de eBird en Argentina y TDF.

Otras especies consideradas raras (Tyto alba y Upucerthia dumetaria) sí se distribuyen hasta TDF, mientras que para el resto (Asthenes pyrrholeuca, Colaptes pitius, Metriopelia melanoptera y Phrygilus fruticeti) no se encontraron referencias que indiquen que su distribución típica incluya a esta provincia, pero fueron registradas en eBird en localidades que se encuentran en paisajes parecidos a los que habitan comúnmente en otras provincias. Esta información, de ser corroborada, podría extender la distribución natural de estas especies incluyendo a la provincia de Tierra del Fuego.

Por otra parte, cabe mencionar que, si bien en los bosques mixtos se ha encontrado el mayor número de especies exclusivas, la totalidad de estas fueron identificadas solo en una o dos observaciones, por lo que fueron consideradas raras. Esto seguramente también esté relacionado con la tendencia de las observaciones.

Aunque eBird ya posee un alcance global, la mayoría de los datos provienen de América del Norte (Sullivan et al., 2014). En dichos países la observación de aves es muy popular, lo que ha generado un aumento y una mejor distribución de los sitios donde se observan aves. En esos sitios, eBird ha sido utilizados mayormente por profesionales, estudiantes y ONG para el estudio de la ecología de las aves, conservación y educación (Lagoze, 2014).

En Argentina, al igual que en otros países de América Latina, las contribuciones a eBird han aumentado en los últimos años y los datos son utilizados como complementos a muestreos $u$ otras bases de datos para estudiar distribuciones geográficas, patrones de movimiento o para incorporar nuevos registros de las aves (Cueto et al., 2011, Salgado-Miranda et al., 2016, Arenas, 2016). En el caso de TDF, Argentina, las observaciones no se encuentran equitativamente repartidas en los distintos puntos de observación, y a su vez, los puntos no se encuentran igualmente distribuidos entre los diferentes paisajes, lo que ocasiona que los resultados del análisis de esta base de datos sean limitados. Es por ello que la estimación de la función ambiental de los bosques empleando las bases de datos de eBird es limitada, pudiendo hacerse pocas inferencias en la comparación entre ambientes boscosos y no boscosos, o entre los distintos tipos de bosque entre sí. Sin embargo, habría que evaluar si la misma es apropiada y confiable para el estudio de los bosques mixtos en sí mismos, ya que se dispone de una mayor cantidad de datos para ellos (por ej., en comparaciones entre el Parque Nacional Tierra del Fuego y la ciudad de Ushuaia). 
Finalmente, cabe mencionar que las aves más frecuentemente observadas en la totalidad de las localidades de cada paisaje se encuentran entre las más conspicuas y conocidas, destacando entre otras probablemente por su carácter manso, poco asustadizo y hábito de uso del estrato inferior del bosque (por ej., C. picta en los paisajes donde predomina la estepa y el bosque de ñire, y $Z$. capensis en bosques de ñire, lenga y mixtos), por su colorido (por ej., S. loyca y $L$. rufa en la estepa), por su tamaño (por ej., T. melanopis en bosques de ñire y M. chimango en bosques de lenga y mixto), o por ser ruidosas (por ej., Caracara plancus en bosques de lenga y mixtos).

Estas diferencias intrínsecas a cada especie también influyen en la posibilidad de detectarlas y en la mayor probabilidad de registro por observadores de aves aficionados, no familiarizados con aquellas especies más silenciosas, pequeñas, poco coloridas o no conspicuas, que son habitantes típicos de estas latitudes.

\section{CONCLUSIONES}

Aunque eBird permitió encontrar diferencias y similitudes entre paisajes, se observa cierta tendencia espacial de concentración de las observaciones, lo que indicaría que son necesarios estudios mejor distribuidos espacialmente y con mayor cantidad de observaciones para caracterizar la avifauna de los paisajes analizados.

A su vez, debido a que en los paisajes de TDF se realizan diferentes actividades que generan impacto, como manejo forestal, ganadería, recreación-turismo y urbanización, son necesarios estudios acerca de la variabilidad interna de esos paisajes.

\section{REFERENCIAS}

Arenas, J., 2016. Registro de la parina chica (Phoenicoparrus jamesi Sclater, 1886) en la Reserva Nacional de Paracas, Perú. Ecología Aplicada, 13 (1).

Bonino, N. y Sbriller, P., 1991. Comparación de las dietas del guanaco, ovino y bovino en Tierra del Fuego, Argentina. Turrialba, 41, 452-452.

Collado, L., 2001. Los bosques de Tierra del Fuego. Análisis de su estratificación mediante imágenes satelitales para el inventario forestal de la provincia. Multequina, 10(1), 15.

Cueto, V. R.; Milesi, F. A.; Sagario, M. C.; Lopez de Casenave, J. y Marone, L., 2011. Distribución geográfica y patrones de movimiento de la Monterita Canela (Poospiza ornata) y el Yal Carbonero (Phrygilus carbonarius) en Argentina. Ornitología Neotropical, 22, 483-494.

Díaz, I. A.; Armesto, J. J.; Reid, S.; Sieving, K. E. and Willson, M. F., 2005. Linking forest structure and composition: Avian diversity in successional forests of Chiloé Island, Chile. Biol. Conservation, 123(1), 91-101.

Gill, F., 2006. Ornithology. W.H. Freeman.

Gottle, A. y Sène, E.H., 1997. Función protectora y ambiental de los recursos forestales. Unasylva 48 (190-191).

Hochachka, W.; Caruana, R.; Fink, D.; Munson, A.; Riedewald, M.; Sorokina, D. and Kelling, S., 2007. Data mining for discovery of pattern and process in ecological systems. Journal of Wildlife Management 71, 24272437.

Humphrey, P. S.; Peterson, D.; Reynolds, R. T. and Humphrey, P. W., 1970. Preliminary Smithsonian Manual: Birds of Isla Grande (Tierra del Fuego) (No. 598.2 PRE).

Ibarra, J. T.; Anderson, C. B.; Altamirano, T. A.; Rozzi, R. and Bonacic, C., 2010. Diversity and singularity of 
avifauna in the austral peat bogs of Cape Horn Biosphere Reserve, Chile. Rev. Ciencia e Investigación Agraria 37:29-43.

Ippi, S.; Anderson, C. B.; Rozzi, R. and Elphick, C. S., 2009. Annual variation of abundance and composition in forest bird assemblages on Navarino Island, Cape Horn Biosphere Reserve, Chile. Ornitologia Neotropical, 20, $231-245$.

Kelling, S., 2008. Significance of organism observations: Data discovery and Access in biodiversity research. Report Presented to the Global Biodiversity Information Facility, Copenhagen.

Lagoze, C., 2014. eBird: Curating citizen science data for use by diverse communities. International Journal of Digital Curation, 9(1), 71-82.

Lencinas M. V.; Gallo E. y Martínez Pastur, G., 2004. Modificación de la biodiversidad por el manejo forestal: Plantas, aves e insectos. Capítulo 1: Sotobosque. Alternativas de Manejo Sustentable para el Manejo Forestal Integral de los Bosques de Patagonia. Módulo, 1.

Lencinas, M. V.; Pastur, G. M.; Medina, M. and Busso, C., 2005. Richness and density of birds in timber Nothofagus pumilio forests and their unproductive associated environments. Biodiversity \& Conservation, 14(10), 2299-2320.

Martínez Pastur, G. J.; Lencinas, M. V.; Gallo, E.; de Cruz, M.; Borla, M. L.; Esteban, R. S. and Anderson, C. B., 2015. Habitat-specific vegetation and seasonal drivers of bird community structure and function in southern Patagonian forests. Community Ecology, 16(1), 55-65.

Meynard, C. N. and Quinn, J. F., 2008. Bird metacommunities in temperate South American forest: Vegetation structure, area, and climate effects. Ecology, 89(4), 981-990.

Michener, W.; Baerwald, T.; Firth, P.; Palmer, M.; Rosenberger, J.; Sandlin, E. and Zimmerman, H., 2001. Defining and unraveling biocomplexity. Bioscience 51, 5.

Montes, C.; De Lamo, D. A. y Zavatti, J., 2000. Distribución de abundancias de guanacos (Lama guanicoe) en los distintos ambientes de Tierra del Fuego, Argentina. Mastozoología Neotropical, 7(1), 5-14.

Moore, D. M., 1983. Flora de Tierra del Fuego. Flora of Tierra del Fuego.

Narosky, T. e Yzurieta, D., 2010. Aves de Argentina y Uruguay. Guía de identificación. Birds of Argentina and Uruguay. A Field Guide. 16a. ed.

Pizarro, J. C.; Anderson, C. B. and Rozzi, R., 2012. Birds as marine-terrestrial linkages in sub-polar archipelagic systems: Avian community composition, function and seasonal dynamics in the Cape Horn Biosphere Reserve (54-55 S), Chile. Polar biology, 35(1), 39-51.

Promis, A.; Cruz, G.; Reif, A. y Gärtner, S., 2008. Bosques de Nothofagus betuloides (Mirb.) Oerdt 1871 (Fagales: Nothofagaceae) en la Patagonia austral y Tierra del Fuego. In Anales del Instituto de la Patagonia (Vol. 36, No. 1, pp. 53-68). Universidad de Magallanes.

Salgado-Miranda, C.; Medina, J. P.; Sánchez-Jasso, J. M. y Soriano-Vargas, E., 2016. Registro altitudinal más alto en México para la cotorra argentina (Myiopsitta monachus). Huitzil, 17(1), 155-159.

Sullivan, B. L.; Wood, C. L.; Iliff, M. J.; Bonney, R. E.; Fink, D. and Kelling, S., 2009. eBird: A citizen-based bird observation network in the biological sciences. Biological Conservation, 142(10), 2282-2292.

Sullivan, B. L.; Aycrigg, J. L.; Barry, J. H.; Bonney, R. E.; Bruns, N.; Cooper, C. B.; Damoulas, T.; Dhondt, A. A.; Dietterich, T.; Farnsworth, A.; Fink, D.; Fitzpatrick, J. W.; Fredericks, T.; Gerbracht, J.; Gomes, C.; Hochachka, W. M.; Iliff, M. J.; Lagoze, C.; La Sorte, F. A.; Merrifield, M.; Morris, W.; Phillips, T. B.; Reynolds, M.; Rodewald, A. D.; Rosenberg, K. V.; Trautmann, N. M.; Wiggins, A.; Winkler, D. W.; Wong, WK.; Wood, C. L.; Yu, J. and Kelling, S., 2014. The eBird enterprise: An integrated approach to development and application of citizen science. Biological Conservation, 169, 31-40. 
